ISSN 1112-9867

Available online at http://www.jfas.info

\title{
GROUNDWATER QUALITY IN ARID REGIONS: THE CASE OF HASSI MESSAOUD REGION (SE ALGERIA)
}

\author{
B. Bouselsal
}

Laboratoire des réservoirs souterrains pétroliers gaziers et aquifères, Faculté des hydrocarbures, des énergies renouvelables et des sciences de la terre. Université de Ouargla

Received: 21 September 2016 / Accepted: 28 December 2016 / Published online: 01 January 2017

\begin{abstract}
After chemical quality study, it has been realized that the groundwater of Hassi Messaoud region isn't drinking one according to WHO and Algerian standards for drinking water. This water is highly mineralized and very hard and its major concentrations are often higher than recommended standards, so it requires treatment before use.

The time that irrigation water quality is based on riverside standards (1954), the waters of the terminal complex of aquifers have average SAR values high, these waters arrange themselves in the poor and bad classes. However Continental Intercalary waters arrange themselves in the poor classes for irrigation.
\end{abstract}

Key words: Terminal Complex, continental intercalary, WHO, potable, Irrigation.

Author Correspondence, e-mail: bousboualem@gmail.com

doi: http://dx.doi.org/10.4314/jfas.v9i1.30

\section{INTRODUCTION}

Dans le Sahara algérien caractérisé par son climat aride, les eaux souterraines représentent la seule source en eaux pour les différents usages. Depuis plus d'une cinquantaine d'années, l'Algérie a commencé l'exploitation du système aquifère de Sahara septentrional (SASS), les prélèvements sont passés de $600 \mathrm{Hm}^{3} /$ an en 1970 [1] à plus que $2000 \mathrm{Hm}^{3} / \mathrm{an}$ en 2015. Malgré que ses eaux souterraines sont abondent mais leur qualité reste toujours un sujet 
actualité nationale et un facteur conditionne le développement démographique et économique du sud algérien [2][3].

Le présent travail est pour objectif de déterminer la qualité chimique des eaux des aquifères du Hassi Messaoud (le complexe terminal et le continental intercalaire), pour l'alimentation en eau potable (AEP) et pour l'irrigation.

\section{PRESENTATION DE LA ZONE D'ETUDE}

La région de Hassi Messaoud (Fig.1) se situe au Sahara septentrional, à $850 \mathrm{Km}$ au SE d'Alger et à $86 \mathrm{~km}$ au sud-est d'Ouargla, limitée par les coordonnées Lambert $\mathrm{X}=$ 790.000/840.000 Est et $\mathrm{Y}=110.000 / 150.000$ Nord. La région compte une population d'enivrent 53000 habitants, elle occupe une superficie d'ordre de $4200 \mathrm{Km}^{2}$, la moitié de cette surface est occupée par des grandes accumulations sableuses d'altitude dépasse $200 \mathrm{~m}$ formant des Ergs. Le climat est hyperaride avec une faible précipitation annuelle (la moyenne est $34.65 \mathrm{~mm}$ ) et une forte température (la moyenne annuelle est $23,5 \mathrm{C}^{\circ}$ ).

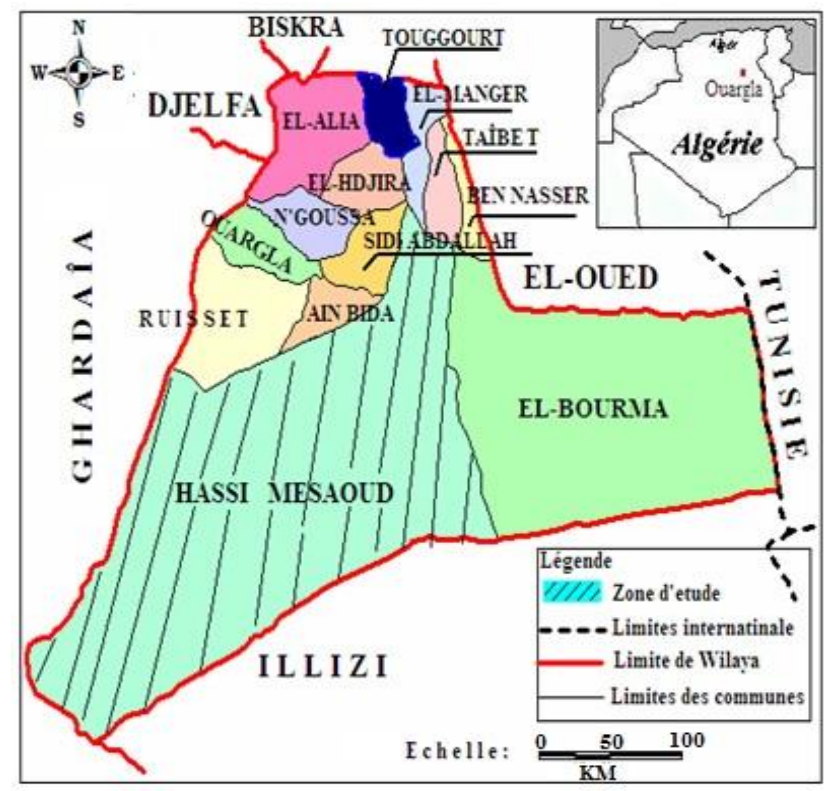

Fig.1. Carte de situation géographique de Hassi Messaoud

La région de Hassi Messouad est une région pétrolière, leur économie est largement tournée vers l'exploitation de son gisement de pétrole, considéré par sa production comme l'un des dix plus grands champs pétroliers au monde, avec actuellement 400000 barils par jour et aurait une durée de vie de 80 ans, il compte plus de mille puits, elle contribue à hauteur de $10 \%$ dans le produit National Brut (PNB). 


\section{CONTEXTE HYDROGEOLOGIQUE.}

Les aquifères de Hassi Messaoud fait partie de système aquifère du Sahara septentrional (SASS) (Fig.2), ce dernier est composé d'une superposition de deux aquifères; le Continental Intercalaire (CI), la plus profonde et celle du Complexe Terminal (CT) [4,5,6,7].

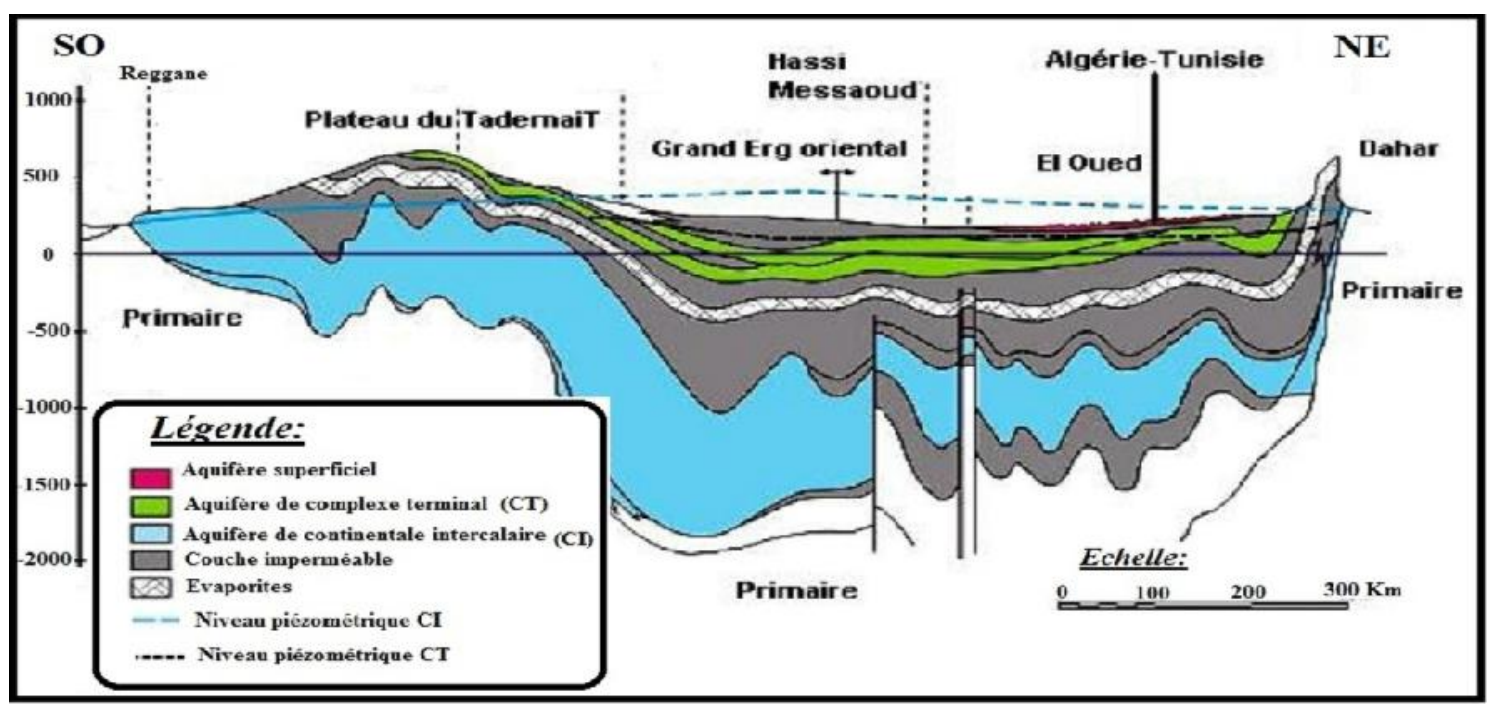

Fig.2. Coupe hydrogéologique dans le Sahara septentrionale (UNESCO 1972)

L'aquifère de continental intercalaire (Fig.3) est constitue de sables et de grés à quelque passées calcaires et d'arrgiles [8,9,10,11] leur profondeur est environ 1150m. La pression de l'eau à la tête de forage est environ 15 bars, le débit varie d'un forage à l'autre, dans les forages pétroliers abandonnés convertis en forages d'eau, il oscille entre 30 et $60 \mathrm{l} / \mathrm{s}$, mais dans les forages d'eau ordinaires, il est d'ordre de100 l/s.

L'aquifère de Complexe Terminal, regroupe deux nappes, de haut en bas on distingue [12], la nappe de Mio-Pliocène constituée de sables avec la présence de calcaire et d'argile, leur épaisseur moyenne est 240m, et la nappe du Sénonien-Eocène formée de calcaire dolomitique et l'anhydrite, d'épaisseur moyenne de $220 \mathrm{~m}$. Le niveau statique de complexe terminal varie entre 18 et 40m et le débit des forages varie entre 15 et 30 1/s.

La nappe du Turonien, constituée de calcaire dolomitique d'épaisseur moyenne de 90m, elle est séparée des deux nappes précédentes par une formation du Sénonien lagunaire qui constitue un écran imperméable. Malgré que cette nappe faite partie de complexe terminal mais en Sahara septentrional algérien, elle est souvent traitée a part parce qu'elle ne présente aucun intérêt économique à cause de sa salinité très élevée (210g/l) [13]. 


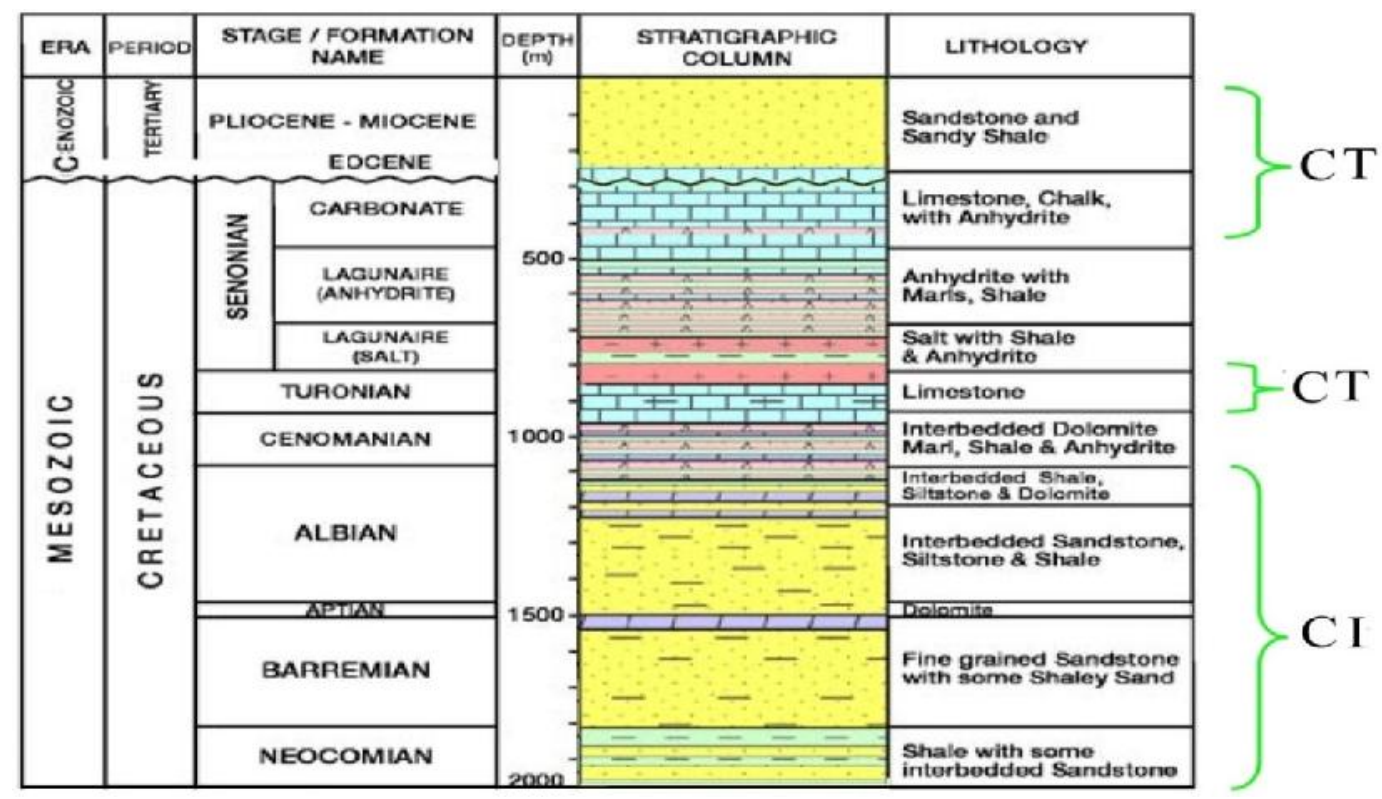

Fig.3. Colonne lithostratigraphique de Hassi Messaoud

Les prélèvements des eaux à partir des aquifères de complexe terminal et de continental intercalaire ne s'asse pas à augmenté chaque année, en 2015, il est estimé à $59 \mathrm{Hm}^{3}$, dont 14 $\mathrm{Hm}^{3}$ pour l'alimentation en eau potable(AEP), $16 \mathrm{Hm}^{3}$ pour l'irrigation et $29 \mathrm{Hm}^{3}$ pour l'industrie. Le volume d'eau exploité pour l'activité pétrolière est estimé à $25 \mathrm{Hm}^{3}$ en 2015 , dont $18 \mathrm{Hm}^{3}$ proviennent du continental intercalaire et $7 \mathrm{Hm}^{3}$ du complexe terminal. Il est utilisé pour l'injection dans les forage pétroliers a fin de maintenir la pression du gisement d'hydrocarbure (65\%), l'alimentation des usines, des bases de vie des sociétés et des chantiers pétroliers (30\%) et les travaux de forage et de work over (5\%). Une partie des forges captant la nappe de continentale intercalaire sont des anciens forages pétroliers abandonnés convertis en forages d'eau. En plus les eaux des nappes salées (turonien et jurassique) sont aussi utilisées pour l'injection dans les forages pétroliers.

\section{MATERIELS ET METHODE}

Les eaux échantillonnées (CT et CI) pour des analyses en éléments majeurs ont été prélevées dans des flacons en verre de $500 \mathrm{ml}$ pour des analyses en anions $\left(\mathrm{Cl}, \mathrm{SO}_{4}, \mathrm{NO}_{3}, \mathrm{~F}\right.$ et $\left.\mathrm{HCO}_{3}\right)$ dissous après filtration, et dans des flacons en verre de $500 \mathrm{ml}$ pour des analyses en cations $(\mathrm{Ca}, \mathrm{Mg}, \mathrm{Na}$ et $\mathrm{K})$ après filtration et acidification.

Le matériel d'échantillonnage a été choisi en fonction des buts de ce travail de recherche. Des outils spéciaux ont été utilisés pendant l'échantillonnage sur le terrain. Pour avoir des résultats sur la qualité des eaux, sur son faciès géochimique (facteurs physico-chimiques), sur sa 
minéralisation vis-à-vis des minéraux dissous et sa potabilité, ont été utilisés les matériels suivants: conductimètre, $\mathrm{pH} / \mathrm{Eh}$ mètre, sonde de température et filtres à $0.45 \mu \mathrm{m}$.

Les analyses des échantillons ont été effectués dans le laboratoire de du Département Techniques Production (IRARA/EP) de Hassi Messaoud, le calcium, magnésium et chlore ont été dosés par titrimétrie. Le sulfate et le fluor par photo-colorimétrie, le sodium et potassium au moyen d'une spectrométrie d'absorption atomique à flamme [14], le nombre d'échantillons analysées est 17 forages captant les deux nappes de complexe terminal et 13 forages captant le continental intercalaire (CI).

\section{RESULTATS ET DISCUSSIONS}

\subsection{Qualité des eaux de complexe terminal}

\subsection{1.Évaluation de la potabilité des eaux}

La qualité des eaux pour l'approvisionnement en eau potable (AEP) ne satisfait pas les normes de l'Organisation Mondiale de la Santé (OMS) [15] et les normes algériennes pour l'eau de boisson. Ainsi, les eaux de complexe terminal de Hassi Messaoud sont inacceptables pour la consommation humaine.

Le Tableau 1 montre la variabilité et l'ordre de grandeur des différents variables physicochimiques caractéristiques des eaux souterraines à l'échelle de tout le secteur étudié, ainsi que les normes de potabilité des eaux de l'Organisation Mondiale de la Santé et les normes algériennes.

Tableau 1: Statistiques élémentaires des différents paramètres physico-chimiques mesurées pour les eaux de complexe terminale.

\begin{tabular}{|c|c|c|c|c|c|c|}
\hline Paramètre & N.ALG & N.OMS & Min & Max & Moy & Ecart-type \\
\hline $\mathrm{Ca}^{+2}(\mathrm{mg} / \mathrm{l})$ & 200 & 200 & 128,00 & 493,30 & 285,26 & 118,28 \\
\hline $\mathrm{Mg}^{+2}(\mathrm{mg} / \mathrm{l})$ & 150 & 150 & 21,60 & 182,00 & 86,90 & 40,24 \\
\hline $\mathrm{Na}^{+}(\mathrm{mg} / \mathrm{l})$ & 200 & 200 & 232,00 & 957,00 & 581,82 & 215,25 \\
\hline $\mathrm{K}^{+}(\mathrm{mg} / \mathrm{l})$ & 20 & 12 & 13,00 & 99,00 & 32,21 & 19,66 \\
\hline $\mathrm{Cl}^{-}(\mathrm{mg} / \mathrm{l})$ & 500 & 250 & 371,00 & 2185,90 & 1017,77 & 501,03 \\
\hline $\mathrm{SO}_{4}^{-2}(\mathrm{mg} / \mathrm{l})$ & 400 & 250 & 400,00 & 1480,00 & 837,64 & 282,01 \\
\hline $\mathrm{HCO}_{3}^{-}$ & - & 300 & 106,00 & 251,00 & 135,98 & 45,14 \\
\hline $\mathrm{F}^{-}(\mathrm{mg} / \mathrm{l})$ & 1,5 & 1,5 & 1,61 & 2,82 & 2,15 & 0,52 \\
\hline $\mathrm{NO}_{3}^{-}(\mathrm{mg} / \mathrm{l})$ & 50 & 50 & 3,26 & 17,43 & 6,42 & 6,71 \\
\hline $\mathrm{Cond}$ & 2800 & 1000 & 2538 & 7383 & 4518 & 1401,6 \\
\hline $\mathrm{pH}$ & $6,5-8,5$ & $6,5-8,5$ & 7,05 & 7,90 & 7,58 & 0,29 \\
\hline
\end{tabular}


La conductivité électrique des eaux de la région varie entre 2538 et $7383 \mu{\mathrm{S} . \mathrm{cm}^{-1}}^{-1}$, avec une valeur moyenne de $4518 \mu \mathrm{S} . \mathrm{cm}^{-1}$. Les eaux à forte conductivité sont celles du puits de CIS

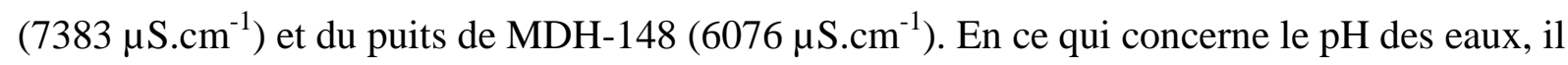
varie entre 7,05 et 7,9, pour une moyenne de 7,58.

La dureté des eaux de CT varie entre $52,8^{\circ} \mathrm{F}$ et $197,3^{\circ} \mathrm{F}$, avec une moyenne de107, $5{ }^{\circ} \mathrm{F}$. La dureté de l'eau est un indicateur du niveau de calcaire dans l'eau, elle correspond à sa teneur en calcium et en magnésium. Plus elle en contient, plus elle est dure. Cela révèle que les eaux de complexe terminale sont très dures.

Un des éléments les plus importants dans l'eau de boisson et lié à la santé est le fluor. Celui-ci se présente dans l'eau sous forme d'ions fluorures $\left(\mathrm{F}^{-}\right)$. Les concentrations en Fluor sont supérieures à $1,5 \mathrm{mg} / \mathrm{l}$, le fluor donc peut occasionner une fluorose dentaire ou osseuse [15, 16, 17,18]. Dans les eaux analysées, les concentrations en $\mathrm{F}^{-}$se situaient entre 1,61 mg/l et $2,82 \mathrm{mg} / \mathrm{l}$. Les ions sulfate $\left(\mathrm{SO}_{4}{ }^{-2}\right)$ à des concentrations supérieures à la valeur guide dans l'eau de boisson peuvent provoquer des diarrhées chez l'être humain [19]. L'analyse de nos données montre que les teneurs en sulfate sont non admissibles, elles dépassent la norme fixée par l'OMS qui est de $250 \mathrm{mg} / \mathrm{l}$ et la norme algérienne de l'eau de boisson qui est de $400 \mathrm{mg} / \mathrm{l}$. La contamination par les nitrates peut être considérée comme acceptable vis-à-vis de la norme $50 \mathrm{mg} / \mathrm{l}$. Les valeurs mesurées varient entre 3,26 mg/l et 17,43 mg/l, avec une valeur moyenne de $6,42 \mathrm{mg} / 1$.

Les concentrations de sodium sont très élevées dépassant la norme de l'OMS et la norme algérienne de l'eau potable (200mg/l), la valeur maximale est de l'ordre de $957 \mathrm{mg} / \mathrm{l}$ mesurée au point OMJH-103 alors que la valeur minimale est de $332 \mathrm{mg} / \mathrm{l}$ mesurée dans le puits OMKH-116. En plus les concentrations de potassium dépassant dans la majorité la norme algérienne de l'eau potable $(20 \mathrm{mg} / \mathrm{l})$, la teneur maximale est de l'ordre de $99 \mathrm{mg} / \mathrm{l}$ alors la valeur minimale est de $13 \mathrm{mg} / \mathrm{l}$, avec une valeur moyenne de $32,21 \mathrm{mg} / \mathrm{l}$.

Les chlorures présentent des teneurs variables et souvent très fortes varient entre $371 \mathrm{mg} / \mathrm{l}$ dans le puits OMOH-112 et 2185,9 mg/l dans le puits CIS. Tous les points de mesure dépassent la norme l'OMS $(250 \mathrm{mg} / \mathrm{l})$ et la norme algérienne de l'eau potable $(500 \mathrm{mg} / \mathrm{l})$ à l'exception de puits OMOH-112.

\subsubsection{Diagramme de PIPER}

Le report de résultats des analyses des eaux de l'aquifère de complexe terminal sur le diagramme triangulaire de Piper [20] (Fig.4), met en évidence l'incidence du faciès lithologique sur la qualité des eaux. Les eaux de l'aquifère de complexe terminal sont 
dominées par les faciès évaporitiques [21, 22, 23], ayant comme origines la dissolution des évaporites (gypse et halite), le mélange avec les eaux de nappe profonde (CI), l'échange de base et les apports anthropiques (pollution). On distingue les facies chimiques suivent:

(a) Le faciès chloruré sodique représente $70,6 \%$ de forages, ces échantillons présentant un rapport $\mathrm{Na} / \mathrm{Cl}$ proche de 1 et un coefficient de corrélation $\left(\mathrm{R}^{2}=0,90\right)$ indiquant une éventuelle dissolution de l'halite et sont caractérisées par une minéralisation élevé (2 à 4,7 g/l).

(b) Le faciès sulfaté sodique représente $23,5 \%$ de forages, ces échantillons résultent de la dissolution de gypse et de l'halite, elles sont caractérisées par une minéralisation moyenne $(1,7$ à $3,4 \mathrm{~g} / \mathrm{l})$.

(c) Le faciès sulfaté calcique représente $5,9 \%$ de forages, ces échantillons présentant un rapport $\mathrm{Ca} / \mathrm{SO}_{4}$ proche de 1 et un coefficient de corrélation $\left(\mathrm{R}^{2}=0,7\right)$ indiquant une éventuelle dissolution de gypse et elles sont caractérisées par une minéralisation faible (1,8 g/l).

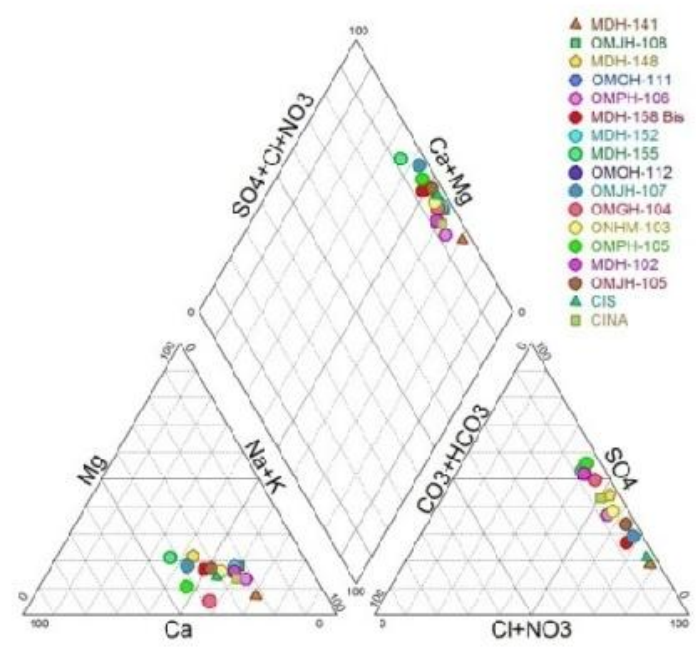

Fig.4. Diagramme de piper des eaux de l'aquifère de CT (juin 2015)

\subsubsection{Qualité des eaux pour usage agricole.}

La qualité d'eau pour l'irrigation, basée sur les normes de Riverside [24], les eaux de complexe terminal de Hassi Messaoud présentent des valeurs de SAR moyennes à fortes (Fig. 5), évoluant dans l'espace avec la conductivité. Ces eaux s'arrangent selon deux classes : 


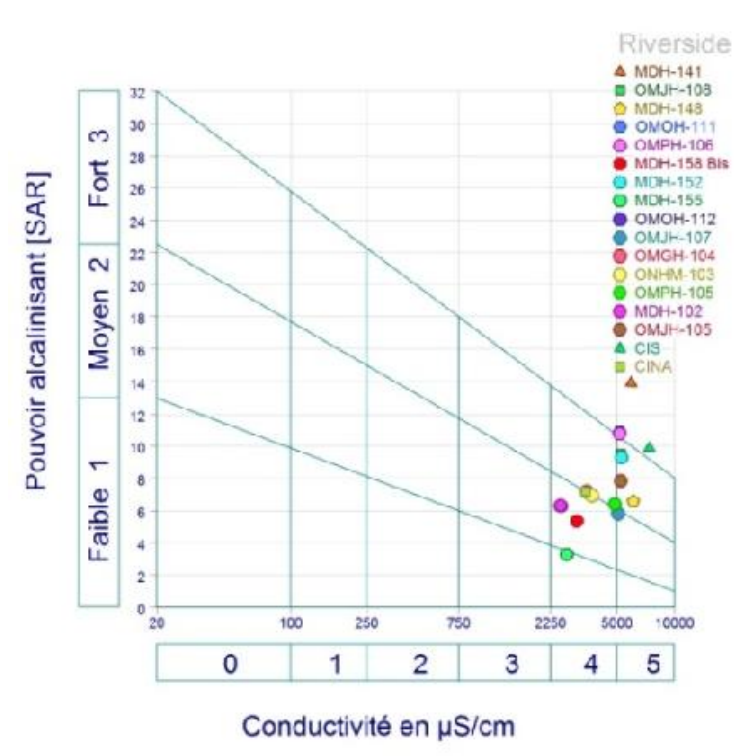

Fig.5. Diagramme de Riverside des eaux de l'aquifère de CT (juin 2015)

(a) eaux médiocres $\left(\mathrm{C}_{4} \mathrm{~S}_{2}-\mathrm{C}_{4} \mathrm{~S}_{1}\right)$ : les eaux faisant partie de cette classe sont fortement chargées en sels, Elles sont susceptibles de convenir à l'irrigation des plantes tolérantes au sel et sur des sols bien drainés et lessivés.

(b) eaux mauvaises $\left(\mathrm{C}_{4} \mathrm{~S}_{3}-\mathrm{C}_{4} \mathrm{~S}_{4}\right)$ : les eaux qui font partie de cette classe ne conviennent pas à l'irrigation, Leur utilisation pour l'irrigation doit être soumise certaines conditions: sols très perméables, bon lessivage, plantes très tolérantes au sel.

\subsection{Qualité des eaux de la nappe continentale intercalaire.}

\subsection{1.Évaluation de la potabilité des eaux.}

La qualité des eaux pour l'approvisionnement en eau potable (AEP) ne satisfait pas les normes de l'Organisation Mondiale de la Santé [15] et les normes algériennes pour l'eau de boisson. Ainsi, les eaux de continental intercalaire de Hassi Messaoud sont inacceptables pour la consommation humaine.

Le tableau 2 montre la variabilité et l'ordre de grandeur des différents paramètres physicochimiques des eaux souterraines à l'échelle de tout le secteur étudié. 
Tableau 2: Statistiques élémentaires des différents paramètres physico-chimiques mesurées pour les eaux de continental intercalaire

\begin{tabular}{|c|c|c|c|c|c|c|}
\hline Paramètre & N.ALG & N.OMS & Min & Max & Moy & Ecart-type \\
\hline $\mathrm{Ca}^{+2}(\mathrm{mg} / \mathrm{l})$ & 200 & 200 & 149,00 & 458,00 & 263,92 & 84,52 \\
\hline $\mathrm{Mg}^{+2}(\mathrm{mg} / \mathrm{l})$ & 150 & 150 & 52,00 & 82,00 & 64,84 & 10,49 \\
\hline $\mathrm{Na}^{+}(\mathrm{mg} / \mathrm{l})$ & 200 & 200 & 270,00 & 440,00 & 343,07 & 48,77 \\
\hline $\mathrm{K}^{+}(\mathrm{mg} / \mathrm{l})$ & 20 & 12 & 28,00 & 90,00 & 53,15 & 17,47 \\
\hline $\mathrm{Cl}^{-}(\mathrm{mg} / \mathrm{l})$ & 500 & 250 & 482,00 & 737,00 & 589,00 & 75,80 \\
\hline $\mathrm{SO}_{4}^{-2}(\mathrm{mg} / \mathrm{l})$ & 400 & 250 & 611,00 & 1031,00 & 770,92 & 119,43 \\
\hline $\mathrm{HCO}_{3}^{-}$ & - & 300 & 110,00 & 299,00 & 193,53 & 50,18 \\
\hline $\mathrm{Cond}$ & 2800 & 1000 & 2955 & 3823 & 3330 & 300 \\
\hline $\mathrm{F}^{-}(\mathrm{mg} / \mathrm{l})$ & 1,5 & 1,5 & 0,62 & 1,18 & 0,82 & 0,23 \\
\hline $\mathrm{NO}_{3}^{-}(\mathrm{mg} / \mathrm{l})$ & 50 & 50 & 0 & 6,6 & 2,8 & 2,5 \\
\hline $\mathrm{pH}$ & $6,5-8,5$ & $6,5-8,5$ & 7,1 & 7,8 & 7,4 & 0,17 \\
\hline
\end{tabular}

Les valeurs de l'écart type dans la plupart des cas sont inférieures à celle de la moyenne ce qui indique une certaine homogénéité dans le type d'eau. Les analyses des paramètres physico-chimiques montrent des valeurs élevées de la conductivité électrique (2955 à 3823 $\mu \mathrm{S} . \mathrm{cm}^{-1}$ ). La température de l'eau de la nappe (CI) est proche de $47^{\circ} \mathrm{C}$ [25], elle dépasse donc la norme de l'eau potable $\left(25^{\circ} \mathrm{C}\right)$. En ce qui concerne le $\mathrm{pH}$ des eaux, il varie entre 7,1 et 7,8, pour une moyenne de 7,49 . La dureté des eaux de CI varie entre $58^{\circ} \mathrm{F}$ et $150^{\circ} \mathrm{F}$, avec une moyenne de $77,5^{\circ} \mathrm{F}$. Cela révèle que les eaux de continental intercalaire sont très dures.

Les concentrations en Fluor sont conformes à la norme de l'OMS et à la norme algérienne pour l'eau de boisson [26], elles se situaient entre $0,62 \mathrm{mg} / \mathrm{l}$ et $1,18 \mathrm{mg} / \mathrm{l}$. même cas pour les nitrates considérés comme acceptable vis-à-vis de la norme, les valeurs mesurées varient entre 0 et $6,6 \mathrm{mg} / \mathrm{l}$, avec une valeur moyenne de $2,8 \mathrm{mg} / \mathrm{l}$.

Les ions de sulfate, de potassium et de sodium dépassent largement, les normes de l'OMS et les normes algériennes. En ce qui concerne les bicarbonates et les chlorures, elles présentent des teneurs variables et souvent dépassent les normes recommandées.

\subsubsection{Diagramme de PIPER.}

La représentation des concentrations en éléments majeurs des eaux de continental intercalaire de la région de Hassi Messaoud sur le diagramme de Piper (Fig.6), met en évidences les faciès chimiques suivant :

(a) Le faciès chloruré sodique $(38,4 \%)$, ces échantillons présentant un rapport $\mathrm{Na} / \mathrm{Cl}$ proche de 1 et un coefficient de corrélation $\left(\mathrm{R}^{2}=0,70\right)$ indiquant une éventuelle dissolution de l'halite. 
(b) Le faciès sulfaté calcique représente $15,4 \%$ de forages, ces échantillons présentant un rapport $\mathrm{Ca} / \mathrm{SO}_{4}$ proche de 1 et un coefficient de corrélation $\left(\mathrm{R}^{2}=0,69\right)$ indiquant une éventuelle dissolution de gypse.

(c) Le faciès sulfaté sodique $(30,8 \%)$ et (d) le faciès chloruré calcique $(15,4 \%)$. Le rapport $\mathrm{SO}_{4} / \mathrm{Cl}$ et le rapport $\mathrm{Na} / \mathrm{Ca}$ montre que, dans la totalité des échantillons les valeurs sont au voisinage de 1 [27], ceci traduit l'influence des matrice de l'Halite et de gypse au même temps.

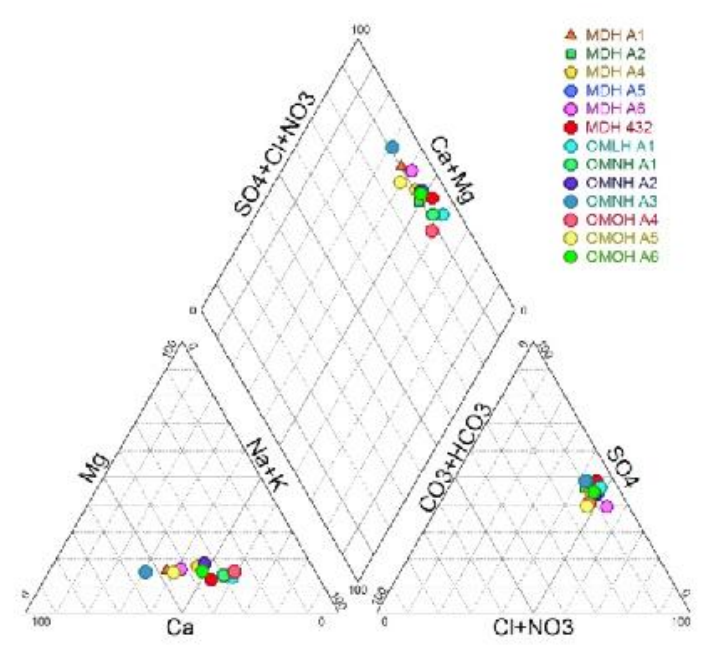

Fig.6. Diagramme de piper des eaux de l'aquifère de CI (juin 2015)

\subsubsection{Qualité des eaux pour usage agricole.}

Le diagramme de Richards (Fig.7) montre que les eaux de la nappe du continentale intercalaire (CI) présentent des valeurs de SAR moyennes, ces eaux s'arrangent dans la classe des eaux médiocres $\left(\mathrm{C}_{4} \mathrm{~S}_{2}\right)$, les eaux faisant partie de cette classe sont fortement chargées en sels, Elles sont susceptibles de convenir à l'irrigation des plantes tolérantes au sel et sur des sols bien drainés et lessivés. 


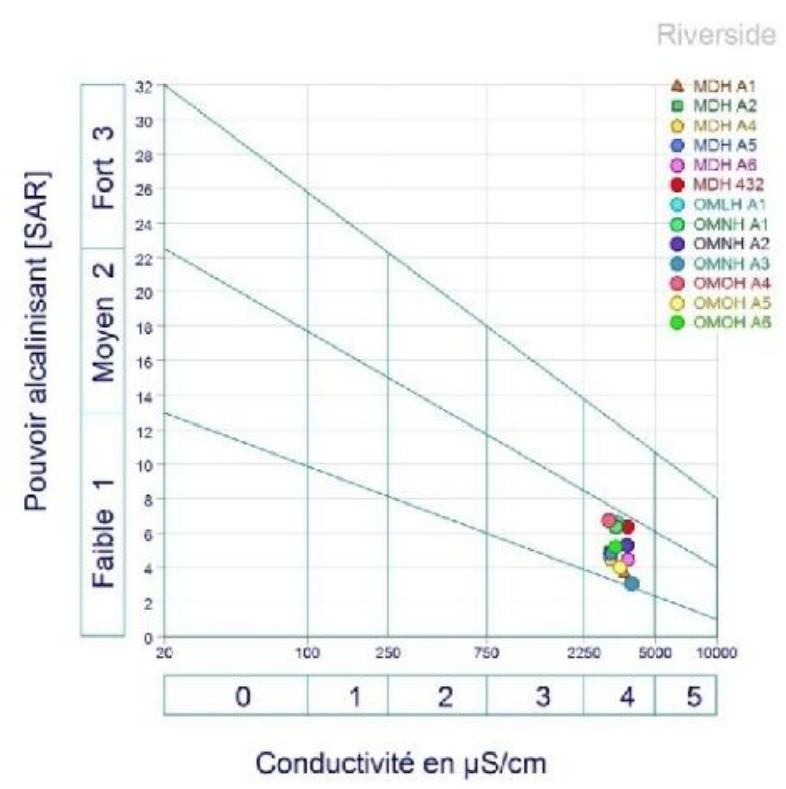

Fig.7. Diagramme de Riverside des eaux de l'aquifère de CT (juin 2015)

\section{CONCLUSION}

La région de Hassi Messaoud, se trouve au Sahara septentrional, c'est une région pétrolière, considéré par sa production comme l'un des dix plus grands champs pétroliers au monde. La sous sol de la région recèle plusieurs aquifères salées et douces, les aquifère appartiens au (SASS) sont les seuls exploités pour les divers usages (l'AEP, l'irrigation et l'industrie), ce sont l'aquifère de complexe terminal (CT) et l'aquifère de continental intercalaire (CI). L'étude de la qualité chimique de ses eaux montre qu'elles sont dominées par les faciès évaporitiques, elles sont non potables en référence aux normes de l'OMS et aux normes algériennes des eaux potables, car elles sont très dures et présentent une minéralisation élevée Elles nécessitent un traitement avant l'utilisation.

Les eaux de complexe terminal présentent une conductivité électrique varie entre 2538 et $7383 \mu \mathrm{S} . \mathrm{cm}^{-1}$, la dureté des eaux varie entre $52,8^{\circ} \mathrm{F}$ et $197,3^{\circ} \mathrm{F}$, les concentrations en Fluor se situaient entre $1,61 \mathrm{mg} / \mathrm{l}$ et $2,82 \mathrm{mg} / \mathrm{l}$. Quant aux concentrations des éléments majeurs sont souvent supérieurs aux normes recommandées.

Les eaux de continental intercalaire présentant des teneures en nitrate et en fluor dans les normes recommandées, mais les teneurs en éléments majeurs de ces eaux sont souvent non conforme aux normes. La conductivité électrique des eaux de CI varie entre 2955 et 3823 $\mu \mathrm{S} . \mathrm{cm}^{-1}$, la température est proche de $47^{\circ} \mathrm{C}$ et la dureté des eaux varie entre $58^{\circ} \mathrm{F}$ et $150^{\circ} \mathrm{F}$.

Quant à la qualité d'eau pour l'irrigation, basée sur les normes de Riverside (1954), les eaux de l'aquifères de complexe terminal présentent des valeurs de SAR moyennes à fortes, ces 
eaux s'arrangent dans les classes médiocre et mauvaise. Pour les eaux Continental Intercalaire, elles s'arrangent dans la classe de $\mathrm{C}_{4} \mathrm{~S}_{2}$ sur le diagramme de Richards, qui désigne des eaux de qualité médiocre pour l'irrigation.

\section{REFERENCES BIBLIOGRAPHIQUES}

[1]OSS (Observatoire du Sahara et du Sahel). 2010. GEOAQUIFER : Amélioration de la connaissance et de la gestion concertée du Système Aquifère du Sahara Septentrional (SASS) par l'utilisation des images satellitaires. Rapport de Synthèse, 23p.

[2]Bouselsal B., Kherici N., Hadj-said S., Belksier M.S. 2014. Qualité des eaux des aquifères de Sahara septentrionale cas: des eaux des aquifères d'el-oued (SE Algérie). International Journal for Environment \& Global Climate Change. Vol 2, Issue 3, 2014. P 21-31

[3]Bouselsal B., Zeddouri A., Belksier M.S., Fenazi B. 2015.Contribution de la Méthode de Vulnérabilité Intrinsèque GOD à l'Etude de la Pollution de la Nappe Libre d'Ouargla (SE Algérie). International Journal for Environment \& Global Climate Change. Vol 3, Issue 4, 2015. P 92-99.

[4]Castany G., 1982. Bassin sédimentaire du Sahara septentrional (Algérie-Tunisie). Aquifères du Continental Intercalaire et du Complexe terminal. Bull. BRGM 2 III (2), 127147.

[5]OSS (Observatoire du Sahara et du Sahel).2003. Système Aquifère du Sahara Septentrional, volume 2 : Hydrogéologie.284p.

[6]Ould baba sy M. 2005. Recharge et paléorecharge du système aquifère du Sahara septentrional. Thèse de Doctorat en Géologie. Faculté des Sciences de Tunis. Tunisie. 277p. [7]UNESCO. (1972). Etude des ressources en eau du Sahara septentrional. Projet ERESS , Rapport final, 7 plaquettes, Paris, France.

[8]ANRH. 2004. Données sur les Logs stratigraphiques des forages de la Wilaya de Ouargla. Algérie. Agence Nationale des Ressources Hydrauliques. Direction régionale Sud, Ouargla.

[9]Busson G. 1970 : Le Mésozoïque saharien. $2^{\text {ème }}$ partie : Essai de synthèse des données des sondages algéro-tunisiens. Edit., Paris, « Centre Rech. Zones Arides », Géol., 11, 811p. Ed. C.N.R.S.

[10]FABRE J. (1976). Introduction à la géologie du Sahara d'Algérie et des régions voisines. SNED, Alger, 142p. 
[11]Edmunds W.M., Guendouz A.H., Mamou A., Moulla A., Shand P., Zouari K. 2003. Groundwater evolution in the Continental Intercalaire aquifer of southern Algeria and Tunisia: trace element and isotopic indicators. Applied Geochemistry 18 (2003) 805-822.

[12]Guendouz A., Moulla A.S., Edmunds W.M., Zouari K., Shands P., Mamou A. 2003. Hydrogeochemical and isotopic evolution of water in the complex terminal aquifer in Algerian Sahara.Hydrogeology Journal. 11. 483-495.

[13]Boudjema A. 2010. Élaboration de la carte de vulnérabilité méthode DRASTIC: (cas de la nappe du Mio-Pliocene a Hassi-Messaoud, Nord-Est Algerien). Communication Science \& technologie, $\mathrm{N}^{\circ}$ 8. Janvier 2010.111-116pp.

[14]Rodier J. 1996. L'analyse de l'eau, eaux naturelles, eaux résiduaires, eau de mer. 8ème édition Dunod, Paris.

[15]OMS (Organisation Mondiale de la Santé). 2004. Directives pour la qualité de l'eau de

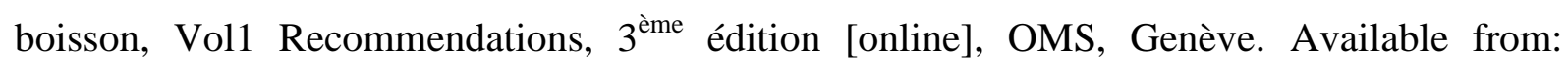
http://www, who, int/entity/water_sanitation_health/dwq/gdwq3_prel_1a5, pdf.

[16]Achour S. 1990. La qualité des eaux du Sahara Septentrional en Algérie. Etude de l'excès en fluor. Tribune de l'eau, Cebedeau, 42 (542), 53-57.

[17]Achour S., Youcef. L. 2001. Excès des fluorures dans les eaux du Sahara septentrional oriental et possibilité de traitement. E.I.N. International, $\mathrm{N}^{\circ} 6$, pp 47-54.

[18]Nezli I.E., Achour S., Djidel M., Attalah S., 2009. Presence and origin of fluoride in the Complex Terminal water of Ouargla basin (Northern Sahara of Algeria). Science Publications. American Journal of Applied Sciences, N06 Mai 2009, pp 876-881.

[19]CDC-EPA. 1999. Health effects from exposure to high levels of sulfate in drinking water study. EPA 815-R-99-001, January 1999.

[20]Piper A.M. 1954. Graphical interpretation of water analysis, Transactions of the American Geophysical Union. 25:914 -928.

[21]Ben Alaya M., Zemni T., Mamou A., Zargouni F. 2014. Acquisition de salinité et qualité des eaux d'une nappe profonde en Tunisie: approche statistique et géochimique. Journal des Sciences Hydrologiques, 59:2,395-419.

[22]Guendouz A., 1985. Contribution a l'étude géochimique et isotopique des nappes profondes du Sahara nord-est septentrional, Algérie. Thèse $3^{\text {ème }}$ cycle, Univ. Paris-Sud, Orsay. [23]Kraiem Z., Zouari K., Bencheikh N., Agoun A., Abidi B. 2015. Processus de minéralisation de la nappe du Plio-Quaternaire dans la plaine de Segui-Zograta (Sud-Ouest tunisien).Hydrological Sciences Journal. 
[24]Richard L.A., 1954. Diagnosis and improvement of saline and alkali soils. Agricultural handbook 60. Washington, DC: USDA, 160p

[25]Idder T., Idder A., Tankari D.A. 2014. «Les oasis du Sahara algérien, entre excédents hydriques et salinité: l'exemple de l'oasis de Ouargla ». Revue des sciences de l'eau / Journal of Water Science, vol. 27, $\mathrm{n}^{\circ} 2,2014$, p. 155-164.

[26]Bouchahm. N., Achour S. 2008. Hydrochimie des eaux souterraines de la région orientale du Sahara septentrional algérien - Identification d'un risque de fluorose endémique. La houille blanche, $\mathrm{N}^{\circ} 02$, pp76-82.

[27]Nezli I.E. 2009. Approche hydrogéochimique a l'étude des aquifères de la basse vallée de l'oued m'ya (Ouargla). Thèse Doctorat, univ.de Biskra, 143 p.

\section{Résumé}

L'étude de la qualité chimique des eaux souterraines de la région de Hassi Messaoud, montre qu'elles sont non potables en référence aux normes de l'OMS et aux normes algériennes des eaux de boisson. Elles sont fortement minéralisées, très dures et les concentrations des éléments majeurs sont souvent supérieures aux normes recommandées. Elles nécessitent un traitement avant l'utilisation

Quant à la qualité d'eau pour l'irrigation, basée sur les normes de Riverside (1954), les eaux de l'aquifère de complexe terminal présentent des valeurs de SAR moyennes à fortes, ces eaux s'arrangent dans les classes médiocre et mauvaise. Pour les eaux de continental Intercalaire, elles s'arrangent dans la classe des eaux médiocre pour l'irrigation.

Mots clés: Complexe Terminal, Continental Intercalaire, OMS, potable, Irrigation.

\section{How to cite this article:}

Bouselsal B. Groundwater quality in arid regions: the case of Hassi Messaoud region (se Algeria). J. Fundam. Appl. Sci., 2017, 9(1), 528-541. 